\title{
NAGATA DIMENSION AND QUASI-MÖBIUS MAPS
}

\author{
XIANGDONG XIE
}

\begin{abstract}
We show that quasi-Möbius maps preserve the Nagata dimension of metric spaces, generalizing a result of U. Lang and T. Schlichenmaier (Int. Math. Res. Not. 2005, no. 58, 3625-3655).
\end{abstract}

\section{INTRODUCTION}

In this note we show that a certain covering dimension of metric spaces - the Nagata dimension - is invariant under quasi-Möbius maps.

Nagata dimension was introduced and studied by Assouad ([A]). It captures both macroscopic and microscopic structures of metric spaces. Hence Nagata dimension has potential applications in both large scale geometry of metric spaces and analysis on metric spaces. S. Buyalo and V. Schroeder ([BS]) have used Nagata dimension to prove that any Gromov hyperbolic group whose Gromov boundary has topological dimension $n$ admits a quasi-isometric embedding into the product of $(n+1)$ trivalent trees. On the other hand, U. Lang and T. Schlichenmaier ([LS]) showed that Nagata dimension is preserved by quasisymmetric maps. Notice that it is clear from the definition (see Section 2) that Nagata dimension is invariant under biLipschitz maps. The purpose of this note is to extend the result of U. Lang and T. Schlichenmaier to quasi-Möbius maps:

Theorem 1.1. Let $f:\left(X_{1}, d_{1}\right) \rightarrow\left(X_{2}, d_{2}\right)$ be a quasi-Möbius map. Then $\left(X_{1}, d_{1}\right)$ and $\left(X_{2}, d_{2}\right)$ have the same Nagata dimension.

The proof of Theorem 1.1 is not difficult. We first observe that each quasi-Möbius map can be written as a composition of a quasisymmetric map and at most two metric inversions (see Section 3 for the definition). Hence by the above mentioned result of U. Lang and T. Schlichenmaier it suffices to show that metric inversions preserve Nagata dimension. This statement is proved by modifying the argument of U. Lang and T. Schlichenmaier (see the proof of Theorem 1.2 in [LS]).

As an immediate application of Theorem 1.1, consider $X=\{1 / n: n=1,2, \cdots\}$ $\subset \mathbb{R}^{2}$ and $Y=\left\{(m, n) \in \mathbb{R}^{2}: m, n \in \mathbb{Z}\right\} \subset \mathbb{R}^{2}$ with the metric induced from $\mathbb{R}^{2}$. Since $X$ has Nagada dimension 1 and $Y$ has Nagata dimension 2, they are not quasi-Möbius equivalent. The author is not aware of a direct proof of this fact.

There are two other dimensions closely related to Nagata dimension: capacity dimension ([B]) and asymptotic dimension of linear type. Capacity dimension concerns the microscopic structure of a metric space and is also invariant

Received by the editors January 23, 2007.

2000 Mathematics Subject Classification. Primary 54F45, 30C65.

Key words and phrases. Nagata dimension, quasi-Möbius maps, metric inversion. 
under quasisymmetric maps. Asymptotic dimension of linear type captures the large scale structure of a metric space and is invariant under quasi-isometries. Notice that capacity dimension is not invariant under quasi-Möbius maps: consider $X=\{1,1 / 2, \cdots, 1 / n, \cdots\} \subset \mathbb{R}^{2}$ and $Y=\{1,2, \cdots, n, \cdots\} \subset \mathbb{R}^{2}$ with the metric induced from $\mathbb{R}^{2}$. The inversion about the unit circle in $\mathbb{R}^{2}$ sends $X$ to $Y$, so $X$ and $Y$ are Möbius equivalent; in particular, they are quasi-Möbius equivalent. However, the capacity dimension of $X$ is 1 while that of $Y$ is 0 .

\section{NAGATA DIMENSION}

In this section we recall some basic facts about Nagata dimension (see [LS], [BL] and $[\mathrm{BDLM}]$ for more details).

Let $(X, d)$ be a metric space and $\mathcal{B}=\left\{B_{i}\right\}_{i \in I}$ a family of subsets of $X$. The family $\mathcal{B}$ is called $D$-bounded, $D \geq 0$, if $\operatorname{diam}\left(B_{i}\right):=\sup \left\{d\left(x, x^{\prime}\right): x, x^{\prime} \in B_{i}\right\} \leq D$ for all $i \in I$. For $s>0$, the $s$-multiplicity of $\mathcal{B}$ is the infimum of all integers $n$ such that every subset of $X$ with diameter $\leq s$ meets at most $n$ members of the family.

Definition 2.1. Let $(X, d)$ be a metric space. The Nagata dimension of $X$, denoted by $\operatorname{dim}_{N}(X, d)$, is the infimum of all integers $n$ with the following property: There exists a constant $c \geq 1$ such that for all $s>0, X$ has a $c s$-bounded covering with $s$-multiplicity at most $n+1$.

Recall that two metric spaces $\left(X_{1}, d_{1}\right)$ and $\left(X_{2}, d_{2}\right)$ are bi-Lipschitz equivalent if there is a bijection $f:\left(X_{1}, d_{1}\right) \rightarrow\left(X_{2}, d_{2}\right)$ and an $L \geq 1$ such that $d_{1}(x, y) / L \leq$ $d_{2}(f(x), f(y)) \leq L d_{1}(x, y)$ holds for all $x, y \in X_{1}$. It is clear from the definitions that two bi-Lipschitz equivalent metric spaces have the same Nagata dimension.

Suppose $s>0$ and $\mathcal{B}$ is a covering of $X$. If $\mathcal{B}$ can be written as a union $\mathcal{B}=\bigcup_{k=0}^{n} \mathcal{B}_{k}$ such that each $\mathcal{B}_{k}$ has $s$-multiplicity at most 1 , then it is clear that $\mathcal{B}$ has $s$-multiplicity at most $n+1$.

If $X=A \cup B$, then $\operatorname{dim}_{N}(X, d)=\max \left\{\operatorname{dim}_{N}(A, d), \operatorname{dim}_{N}(B, d)\right\}$ (Theorem 2.7 of [LS]). In particular, if $X$ contains at least two points and $p \in X$, then $\operatorname{dim}_{N}(X, d)=\operatorname{dim}_{N}(X \backslash\{p\}, d)$ (since the Nagata dimension of a singleton is 0 ).

A key ingredient in the proof of Theorem 1.1 is the following existence result of a sequence of coverings. There are similar results for asymptotic dimension ([D], Proposition 1) and capacity dimension ([B], Proposition 4.4). Notice that the existence of a sequence of coverings as in Proposition $2.2 \operatorname{implies}^{\operatorname{dim}_{N}}(X, d) \leq n$. We shall only use properties (i)-(iii).

Proposition 2.2 (Proposition 4.1 of $[\mathrm{LS}]$ ). Suppose $(X, d)$ is a metric space with $\operatorname{dim}_{N}(X, d) \leq n<\infty$. Then there is a constant $c \geq 1$ such that for all sufficiently large $r>1$, there exists a sequence of coverings $\mathcal{B}^{j}$ of $X, j \in \mathbb{Z}$, with the following properties:

(i) For every $j \in \mathbb{Z}$, we have $\mathcal{B}^{j}=\bigcup_{k=0}^{n} \mathcal{B}_{k}^{j}$ where each $\mathcal{B}_{k}^{j}$ is a cr ${ }^{j}$-bounded family with $r^{j}$-multiplicity at most 1.

(ii) For all $j \in \mathbb{Z}$ and $x \in X$, there exists a $C \in \mathcal{B}^{j}$ that contains the closed ball $B\left(x, r^{j}\right)$.

(iii) Whenever $B \in \mathcal{B}_{k}^{i}$ and $C \in \mathcal{B}_{k}^{j}$ for some $k$ and $i<j$, then either $B \subset C$ or $d(x, y) \geq r^{i}$ for every pair of points $x \in B, y \in C$.

(iv) For every $k \in\{0,1, \cdots, n\}$ and every bounded set $B \subset X$, there is a $C \in$ $\mathcal{B}_{k}:=\bigcup_{j \in \mathbb{Z}} \mathcal{B}_{k}^{j}$ such that $B \subset C$. 


\section{QUASI-MÖBIUS MAPS AND METRIC INVERSIONS}

In this Section we recall the notions of quasisymmetric maps, quasi-Möbius maps and metric inversions, and discuss their relations (see [BHX], [V], [H] and [V2] for more details).

Let $\eta:[0, \infty) \rightarrow[0, \infty)$ be a homeomorphism. A homeomorphism between metric spaces $f:\left(X, d_{X}\right) \rightarrow\left(Y, d_{Y}\right)$ is $\eta$-quasisymmetric if for all pairwise distinct points $x, y, z \in X$, we have

$$
\frac{d_{Y}(f(x), f(y))}{d_{Y}(f(x), f(z))} \leq \eta\left(\frac{d_{X}(x, y)}{d_{X}(x, z)}\right) .
$$

A homeomorphism $f:\left(X, d_{X}\right) \rightarrow\left(Y, d_{Y}\right)$ is quasisymmetric if it is $\eta$-quasisymmetric for some $\eta$. Quasisymmetric maps send bounded metric spaces to bounded metric spaces (Proposition 10.8 of $[\mathrm{H}]$ ).

Let $Q=\left(x_{1}, x_{2}, x_{3}, x_{4}\right)$ be a quadruple of pairwise distinct points in $(X, d)$. The cross ratio of $Q$ with respect to the metric $d$ is:

$$
\operatorname{cr}(Q, d)=\frac{d\left(x_{1}, x_{3}\right) d\left(x_{2}, x_{4}\right)}{d\left(x_{1}, x_{4}\right) d\left(x_{2}, x_{3}\right)} .
$$

Let $\eta:[0, \infty) \rightarrow[0, \infty)$ be a homeomorphism. A homeomorphism between metric spaces $f:\left(X, d_{X}\right) \rightarrow\left(Y, d_{Y}\right)$ is an $\eta$-quasi-Möbius map if

$$
\operatorname{cr}\left(f(Q), d_{Y}\right) \leq \eta\left(\operatorname{cr}\left(Q, d_{X}\right)\right)
$$

for all quadruple $Q$ of distinct points in $X$, where $f(Q)=\left(f\left(x_{1}\right), f\left(x_{2}\right), f\left(x_{3}\right), f\left(x_{4}\right)\right)$. A homeomorphism $f:\left(X, d_{X}\right) \rightarrow\left(Y, d_{Y}\right)$ is quasi-Möbius if it is $\eta$-quasi-Möbius for some $\eta$. The inverse of a quasi-Möbius map is quasi-Möbius, and the composition of two quasi-Möbius maps is also quasi-Möbius (see [V]).

Quasisymmetric maps are quasi-Möbius. In general quasi-Möbius maps are not quasisymmetric; for example, the inversions about spheres in Euclidean spaces are Möbius (hence quasi-Möbius) but are not quasisymmetric. However, quasi-Möbius maps between bounded metric spaces are quasisymmetric (see [V]). Below we will find further connection between quasisymmetric maps and quasi-Möbius maps (Proposition 3.6). We first recall the notion of metric inversion, which is a generalization of inversions about unit spheres in Euclidean spaces.

Let $(X, d)$ be a metric space and $p \in X$. Set $I_{p}(X)=X \backslash\{p\}$ if $X$ is bounded and $I_{p}(X)=(X \backslash\{p\}) \cup\{\infty\}$ if $X$ is unbounded, where $\infty$ is a point not in $X$. Then there is a metric $d_{p}$ on $I_{p}(X)$ such that the following holds for all $x_{1}, x_{2} \in X \backslash\{p\}$ :

$$
\frac{d\left(x_{1}, x_{2}\right)}{4 d\left(x_{1}, p\right) d\left(x_{2}, p\right)} \leq d_{p}\left(x_{1}, x_{2}\right) \leq \frac{d\left(x_{1}, x_{2}\right)}{d\left(x_{1}, p\right) d\left(x_{2}, p\right)} .
$$

When $X$ is unbounded, the following also holds for all $x \in X \backslash\{p\}$ :

$$
\frac{1}{4 d(x, p)} \leq d_{p}(x, \infty) \leq \frac{1}{d(x, p)}
$$

Furthermore, the identity map id $:(X \backslash\{p\}, d) \rightarrow\left(X \backslash\{p\}, d_{p}\right)$ is an $\eta$-quasi-Möbius homeomorphism with $\eta(t)=16 t$ (see [BHX] for a proof of the above statements). For any metric space $(X, d)$ and $p \in X$, we call the identity map id $:(X \backslash\{p\}, d) \rightarrow$ $\left(X \backslash\{p\}, d_{p}\right)$ a metric inversion. 
Lemma 3.2. Let $(X, d)$ be a metric space, $p \in X$ and $A \subset X \backslash\{p\}$ a subset. Suppose there are positive numbers $R>r>0$ such that $r \leq d(x, p) \leq R$ for all $x \in A$. Then the identity map id $:(A, d) \rightarrow\left(A, d_{p}\right)$ is a bi-Lipschitz map.

Proof. Let $x, y \in A$. By the assumption $r \leq d(x, p), d(y, p) \leq R$. Hence $d_{p}(x, y) \leq$ $\frac{d(x, y)}{d(x, p) d(y, p)} \leq \frac{1}{r^{2}} d(x, y)$ and $d_{p}(x, y) \geq \frac{d(x, y)}{4 d(x, p) d(y, p)} \geq \frac{1}{4 R^{2}} d(x, y)$.

The next two lemmas say that the composition of two suitable metric inversions is bi-Lipschitz.

Let $(X, d)$ be an unbounded metric space and $p \in X$. Then $d_{p}$ is a metric on $I_{p}(X)=(X \backslash\{p\}) \cup\{\infty\}$. Set $Y=I_{p}(X)$ and $\rho=d_{p}$. Then $\rho_{\infty}$ is a metric on $Y \backslash\{\infty\}=X \backslash\{p\}$.

Lemma 3.3 (Proposition 3.7 of $[\mathrm{BHX}])$. Let $(X, d)$ and $\rho_{\infty}$ be as above. Then the identity map id $:(X \backslash\{p\}, d) \rightarrow\left(X \backslash\{p\}, \rho_{\infty}\right)$ is 16-bi-Lipschitz.

We next describe a procedure which is analogous to obtaining the chordal metric on $\mathbb{S}^{2}$ from the standard metric on $\mathbb{R}^{2}$ via inverse stereographic projection.

Let $(Y, \rho)$ be an unbounded metric space and $q \in Y$. It is shown in [BK] (Lemma 2.2) that there is a metric $\rho^{q}$ on $Y$ such that for all $y_{1}, y_{2} \in Y$,

$$
\frac{\rho\left(y_{1}, y_{2}\right)}{4\left(1+\rho\left(y_{1}, q\right)\right)\left(1+\rho\left(y_{2}, q\right)\right)} \leq \rho^{q}\left(y_{1}, y_{2}\right) \leq \frac{\rho\left(y_{1}, y_{2}\right)}{\left(1+\rho\left(y_{1}, q\right)\right)\left(1+\rho\left(y_{2}, q\right)\right)}
$$

The metric $\rho^{q}$ can be described as follows. Let $Z=Y \cup\left\{q^{\prime}\right\}$ be the disjoint union of $Y$ and a point $q^{\prime}$. Define a metric $\rho^{\prime}$ on $Z$ by: $\rho^{\prime}\left(q^{\prime}, q^{\prime}\right)=0, \rho^{\prime}\left(y, q^{\prime}\right)=1+\rho(y, q)$ for $y \in Y$ and $\rho^{\prime}\left(y_{1}, y_{2}\right)=\rho\left(y_{1}, y_{2}\right)$ for $y_{1}, y_{2} \in Y$. Set $\rho^{q}=\rho_{q^{\prime}}^{\prime}$. Then, by the paragraph preceding Lemma 3.2, $\rho^{q}$ satisfies $(3.4)$, and the identity map $(Y, \rho) \rightarrow$ $\left(Y, \rho^{q}\right)$ is precisely the metric inversion $\left(Z \backslash\left\{q^{\prime}\right\}, \rho^{\prime}\right) \rightarrow\left(Z \backslash\left\{q^{\prime}\right\}, \rho_{q^{\prime}}^{\prime}\right)$. The second inequality in (3.4) also implies $\operatorname{diam}\left(Y, \rho^{q}\right) \leq 1$. In particular, $\left(Y, \rho^{q}\right)$ is bounded.

Now let $(X, d)$ be a bounded metric space, $p \in X$ a non-isolated point, and $q \in X \backslash\{p\}$. Then the metric space $\left(X \backslash\{p\}, d_{p}\right)$ is unbounded, and so we may form the bounded metric space $\left(X \backslash\{p\},\left(d_{p}\right)^{q}\right)$.

Lemma 3.5. Let $(X, d)$ be a bounded metric space, $p \in X$ a non-isolated point, and $q \in X \backslash\{p\}$. Then the identity map id $:(X \backslash\{p\}, d) \rightarrow\left(X \backslash\{p\},\left(d_{p}\right)^{q}\right)$ is bi-Lipschitz.

Proof. Set $a=\min \left\{1, \frac{1}{4 d(q, p)}\right\}, b=\max \left\{1, \frac{1}{d(q, p)}\right\}$ and $c=\operatorname{diam}(X, d)$. Let $x_{1}, x_{2} \in$ $X \backslash\{p\}$. Then

$$
\begin{aligned}
\left(d_{p}\right)^{q}\left(x_{1}, x_{2}\right) & \leq \frac{d_{p}\left(x_{1}, x_{2}\right)}{\left(1+d_{p}\left(x_{1}, q\right)\right)\left(1+d_{p}\left(x_{2}, q\right)\right)} \\
& \leq \frac{d\left(x_{1}, x_{2}\right)}{d\left(x_{1}, p\right) d\left(x_{2}, p\right)}\left(1+\frac{d\left(x_{1}, q\right)}{4 d\left(x_{1}, p\right) d(q, p)}\right)^{-1}\left(1+\frac{d\left(x_{2}, q\right)}{4 d\left(x_{2}, p\right) d(q, p)}\right)^{-1} \\
& =d\left(x_{1}, x_{2}\right)\left(d\left(x_{1}, p\right)+\frac{d\left(x_{1}, q\right)}{4 d(q, p)}\right)^{-1}\left(d\left(x_{2}, p\right)+\frac{d\left(x_{2}, q\right)}{4 d(q, p)}\right)^{-1} \\
& \leq d\left(x_{1}, x_{2}\right) \cdot a^{-2}\left(d\left(x_{1}, p\right)+d\left(x_{1}, q\right)\right)^{-1}\left(d\left(x_{2}, p\right)+d\left(x_{2}, q\right)\right)^{-1} \\
& \leq \frac{1}{a^{2} d^{2}(p, q)} d\left(x_{1}, x_{2}\right) .
\end{aligned}
$$


Similarly we have

$$
\begin{aligned}
\left(d_{p}\right)^{q}\left(x_{1}, x_{2}\right) & \geq \frac{1}{16 b^{2}\left(d\left(x_{1}, p\right)+d\left(x_{1}, q\right)\right)\left(d\left(x_{2}, p\right)+d\left(x_{2}, q\right)\right)} d\left(x_{1}, x_{2}\right) \\
& \geq \frac{1}{16 b^{2} \cdot 2 c \cdot 2 c} d\left(x_{1}, x_{2}\right) \\
& =\frac{1}{64 b^{2} c^{2}} d\left(x_{1}, x_{2}\right) .
\end{aligned}
$$

Proposition 3.6. Let $f:\left(X_{1}, d_{1}\right) \rightarrow\left(X_{2}, d_{2}\right)$ be a quasi-Möbius homeomorphism. Then $f$ can be written as $f=f_{2}^{-1} \circ f^{\prime} \circ f_{1}$, where $f^{\prime}$ is a quasisymmetric map, and $f_{i}(i=1,2)$ is either a metric inversion or the identity map on the metric space $\left(X_{i}, d_{i}\right)$.

Proof. Let $i \in\{1,2\}$. If $\left(X_{i}, d_{i}\right)$ is bounded, we let $\rho_{i}=d_{i}$ and $f_{i}=\mathrm{id}:\left(X_{i}, d_{i}\right) \rightarrow$ $\left(X_{i}, \rho_{i}\right)$ be the identity map on the metric space $\left(X_{i}, d_{i}\right)$. If $\left(X_{i}, d_{i}\right)$ is unbounded, then we pick $q_{i} \in X_{i}$ and set $\rho_{i}=\left(d_{i}\right)^{q_{i}}$ and let $f_{i}:\left(X_{i}, d_{i}\right) \rightarrow\left(X_{i}, \rho_{i}\right)$ be the identity map (of the set $X_{i}$ ). In this case, $f_{i}$ is a metric inversion (on an auxiliary space) by our discussion in the second paragraph after Lemma 3.3. Set $f^{\prime}:=f_{2} \circ f \circ f_{1}^{-1}:\left(X_{1}, \rho_{1}\right) \rightarrow\left(X_{2}, \rho_{2}\right)$. Then $f=f_{2}^{-1} \circ f^{\prime} \circ f_{1}$. Since $f_{1}, f_{2}$ and $f$ are quasi-Möbius maps, $f^{\prime}$ is also quasi-Möbius. The fact that $\left(X_{1}, \rho_{1}\right)$ and $\left(X_{2}, \rho_{2}\right)$ are bounded metric spaces now implies that $f^{\prime}$ is actually a quasisymmetric map.

\section{Proof of the MAIN THEOREM}

In this Section we prove Theorem 1.1. By Proposition 3.6, Theorem 1.1 follows from the following two results.

Theorem 4.1 (Theorem 1.2 of $[\mathrm{LS}])$. Suppose $\left(X_{1}, d_{1}\right)$ and $\left(X_{2}, d_{2}\right)$ are quasisymmetric, then $\operatorname{dim}_{N}\left(X_{1}, d_{1}\right)=\operatorname{dim}_{N}\left(X_{2}, d_{2}\right)$.

Theorem 4.2. Let $(X, d)$ be a metric space and $p \in X$. Then $\operatorname{dim}_{N}(X \backslash\{p\}, d)=$ $\operatorname{dim}_{N}\left(X \backslash\{p\}, d_{p}\right)$.

To prove Theorem 4.2, we need:

Proposition 4.3. Let $(X, d)$ be a metric space and $p \in X$. Then $\operatorname{dim}_{N}(X \backslash\{p\}, d) \geq$ $\operatorname{dim}_{N}\left(X \backslash\{p\}, d_{p}\right)$.

Assuming Proposition 4.3, we first finish the proof of Theorem 4.2.

Proof of Theorem 4.2. We first suppose that $(X, d)$ is unbounded. Pick $p \in X$. Then $d_{p}$ is a metric on $I_{p}(X)=(X \backslash\{p\}) \cup\{\infty\}$. Set $Y=I_{p}(X)$ and $\rho=d_{p}$. Then $\rho_{\infty}$ is a metric on $Y \backslash\{\infty\}=X \backslash\{p\}$. Proposition 4.3 applied to $(X \backslash\{p\}, d) \rightarrow$ $\left(X \backslash\{p\}, d_{p}\right)$ and $\left(X \backslash\{p\}, d_{p}\right)=(Y \backslash\{\infty\}, \rho) \rightarrow\left(Y \backslash\{\infty\}, \rho_{\infty}\right)=\left(X \backslash\{p\}, \rho_{\infty}\right)$ implies $\operatorname{dim}_{N}(X \backslash\{p\}, d) \geq \operatorname{dim}_{N}\left(X \backslash\{p\}, d_{p}\right) \geq \operatorname{dim}_{N}\left(X \backslash\{p\}, \rho_{\infty}\right)$. On the other hand, Lemma 3.3 implies $\operatorname{dim}_{N}(X \backslash\{p\}, d)=\operatorname{dim}_{N}\left(X \backslash\{p\}, \rho_{\infty}\right)$. The theorem follows in this case.

Next we suppose that $(X, d)$ is bounded. Pick $p \neq q \in X$. If $p$ is isolated, then Lemma 3.2 implies that the metric inversion $(X \backslash\{p\}, d) \rightarrow\left(X \backslash\{p\}, d_{p}\right)$ is biLipschitz and hence $\operatorname{dim}_{N}(X \backslash\{p\}, d)=\operatorname{dim}_{N}\left(X \backslash\{p\}, d_{p}\right)$. So we assume that $p$ is a non-isolated point. Then the metric space $\left(X \backslash\{p\}, d_{p}\right)$ is unbounded, and so we 
may form the bounded metric space $\left(X \backslash\{p\},\left(d_{p}\right)^{q}\right)$. By our discussion in the second paragraph after Lemma 3.3, the identity map $\left(X \backslash\{p\}, d_{p}\right) \rightarrow\left(X \backslash\{p\},\left(d_{p}\right)^{q}\right)$ is a metric inversion on an auxiliary space. Proposition $4.3 \operatorname{implies}_{\operatorname{dim}_{N}}(X \backslash\{p\}, d) \geq$ $\operatorname{dim}_{N}\left(X \backslash\{p\}, d_{p}\right) \geq \operatorname{dim}_{N}\left(X \backslash\{p\},\left(d_{p}\right)^{q}\right)$. Now the theorem follows from Lemma 3.5.

The rest of the section is devoted to the proof of Proposition 4.3.

We may assume that $X$ contains at least two points and $n:=\operatorname{dim}_{N}(X \backslash\{p\}, d)<$ $\infty$. Apply Proposition 2.2. Then there exist $c \geq 1, r>1$, and a sequence of coverings $\mathcal{B}^{j}$ of $(X \backslash\{p\}, d), j \in \mathbb{Z}$, with properties (i)-(iii) as stated in Proposition 2.2. Set $\bar{c}=16 c r$ and $c^{\prime}=640 \bar{c}(\bar{c}+1)$. We shall prove that for each $s>0,\left(X \backslash\{p\}, d_{p}\right)$ has a $c^{\prime} s$-bounded covering with $s$-multiplicity at most $n+1$.

Set $a=\inf \{d(x, p): x \in X \backslash\{p\}\}$ and $b=\sup \{d(x, p): x \in X \backslash\{p\}\}$. Then $0 \leq a \leq b \leq \infty$. We may assume that either $a=0$ or $b=\infty$ holds, for otherwise Lemma 3.2 implies that id $:(X \backslash\{p\}, d) \rightarrow\left(X \backslash\{p\}, d_{p}\right)$ is bi-Lipschitz and hence $\operatorname{dim}_{N}\left(X \backslash\{p\}, d_{p}\right)=\operatorname{dim}_{N}(X \backslash\{p\}, d)=n$.

If $a>0, b=\infty$, and $s \geq(320 \bar{c}(\bar{c}+1) a)^{-1}$, then

$$
d_{p}(x, y) \leq \frac{d(x, y)}{d(x, p) d(y, p)} \leq \frac{1}{d(x, p)}+\frac{1}{d(y, p)} \leq \frac{2}{a}
$$

for all $x, y \in X \backslash\{p\}$. Hence $\operatorname{diam}\left(X \backslash\{p\}, d_{p}\right) \leq 2 / a \leq c^{\prime} s$. In this case the family $\{X \backslash\{p\}\}$ consisting of one element is a $c^{\prime} s$-bounded covering of $\left(X \backslash\{p\}, d_{p}\right)$.

From now on we assume $s$ satisfies $0<s<(320 \bar{c}(\bar{c}+1) a)^{-1}$ (which is simply $s>0$ if $a=0)$. Set $A_{s}=\left\{x \in X: 0<d(x, p) \leq(160 \bar{c}(\bar{c}+1) s)^{-1}\right\}$. Notice that $(160 \bar{c}(\bar{c}+1) s)^{-1}>a$. Hence $A_{s}$ is nonempty by the definition of $a$.

Let $x \in A_{s}$. By property (ii), for each $j \in \mathbb{Z}$, there is some $C \in \mathcal{B}^{j}$ with $B\left(x, r^{j}\right) \subset C$. By property (i), $\operatorname{diam}(C, d) \leq c r^{j}$. It follows from inequality (3.1) that $\operatorname{diam}\left(C, d_{p}\right) \rightarrow 0$ as $j \rightarrow-\infty$.

Lemma 4.4. Let $x \in A_{s}$ and set

$$
j(x):=\sup \left\{j \in \mathbb{Z}: \text { there is } C \in \mathcal{B}^{j} \text { with } B\left(x, r^{j}\right) \subset C \text { and } \operatorname{diam}\left(C, d_{p}\right) \leq \bar{c} s\right\} .
$$

Then $j(x)<\infty$.

Proof. First suppose $a=0$. In this case there is a sequence $x_{i} \in X \backslash\{p\}$ with $d\left(p, x_{i}\right) \rightarrow 0$. Let $j \in \mathbb{Z}$ satisfy $r^{j}>d(x, p)$. Then $x_{i} \in B\left(x, r^{j}\right)$ for all sufficiently large $i$. Since $d_{p}\left(x, x_{i}\right) \geq \frac{d\left(x, x_{i}\right)}{4 d(x, p) d\left(x_{i}, p\right)} \rightarrow \infty$ as $i \rightarrow \infty$, we have $\operatorname{diam}\left(B\left(x, r^{j}\right), d_{p}\right)=$ $\infty$. It follows that $j(x)$ satisfies $r^{j(x)} \leq d(x, p)$. In particular, $j(x)<\infty$.

Now assume $b=\infty$. In this case, there is a sequence of points $x_{i} \in X$ with $d\left(x_{i}, p\right) \rightarrow \infty$. Fix some index $i_{0}$ such that $\frac{d\left(x, x_{i_{0}}\right)}{d\left(x_{i_{0}}, p\right)} \geq 1 / 2$. Let $j \in \mathbb{Z}$ satisfy $r^{j}>d\left(x, x_{i_{0}}\right)$. Then $x_{i_{0}} \in B\left(x, r^{j}\right)$ and so

$\operatorname{diam}\left(B\left(x, r^{j}\right), d_{p}\right) \geq d_{p}\left(x, x_{i_{0}}\right) \geq \frac{d\left(x, x_{i_{0}}\right)}{4 d(x, p) d\left(x_{i_{0}}, p\right)} \geq \frac{1}{8 d(x, p)} \geq 160 \bar{c}(\bar{c}+1) s / 8>\bar{c} s$.

It follows that $j(x)$ satisfies $r^{j(x)} \leq d\left(x, x_{i_{0}}\right)$. In particular, $j(x)<\infty$.

By Lemma 4.4, for each $x \in A_{s}$, we can fix some $C_{x} \in \mathcal{B}^{j(x)}$ that satisfies $B\left(x, r^{j(x)}\right) \subset C_{x}$ and $\operatorname{diam}\left(C_{x}, d_{p}\right) \leq \bar{c} s$. Consider the subfamily of $\mathcal{B}: \mathcal{C}:=$ $\left\{C_{x} \in \mathcal{B}: x \in A_{s}\right\}$. 
Lemma 4.5. For every member $C \in \mathcal{C}$, there exists a maximal element $C^{\prime}$ of $\mathcal{C}$ with respect to inclusion, such that $C \subset C^{\prime}$.

Proof. It suffices to show that there is no infinite strictly increasing sequence in $\mathcal{C}$. Suppose there is a sequence $\left\{C_{x_{i}}\right\}_{i=1}^{\infty} \subset \mathcal{C}$ such that $C_{x_{i}}$ is strictly contained in $C_{x_{i+1}}$ for all $i \geq 1$. Since $\mathcal{C} \subset \mathcal{B}$ and $\mathcal{B}=\bigcup_{k=0}^{n} \mathcal{B}_{k}$, after passing to a subsequence, we may assume that there is some $k \in\{0,1, \cdots, n\}$ such that $C_{x_{i}} \in \mathcal{B}_{k}$ for all $i$. Property (i) of Proposition 2.2 implies that $j\left(x_{i}\right) \neq j\left(x_{l}\right)$ whenever $i \neq l$. After passing to a further subsequence, we may assume that $j\left(x_{i}\right) \rightarrow+\infty$ or $j\left(x_{i}\right) \rightarrow-\infty$ as $i \rightarrow \infty$. Recall that $\mathcal{B}^{j}$ is $c r^{j}$-bounded. If $j\left(x_{i}\right) \rightarrow-\infty$, then $C_{x_{i}}=\left\{x_{i}\right\}$ and $x_{i}=x_{l}$ for all $i, l$, contradicting the assumption that $C_{x_{i}} \neq C_{x_{i+1}}$. Hence we must have $j\left(x_{i}\right) \rightarrow+\infty$. Since all $x_{i}$ lie in the bounded set $A_{s}$ we have $\bigcup_{i=1}^{\infty} C_{x_{i}}=X \backslash\{p\}$. By the definition of $j(x), \operatorname{diam}\left(C_{x_{i}}, d_{p}\right) \leq \bar{c} s$. Hence $\operatorname{diam}\left(X \backslash\{p\}, d_{p}\right) \leq \bar{c} s$. If $a=0$, then $\left(X \backslash\{p\}, d_{p}\right)$ is unbounded. Hence we must have $b=\infty$. Pick $y_{l} \in X$ with $d\left(y_{l}, p\right) \rightarrow \infty$. Then for any $x \in A_{s}$, we have

$$
d_{p}\left(x, y_{l}\right) \geq \frac{d\left(x, y_{l}\right)}{4 d(x, p) d\left(y_{l}, p\right)} \rightarrow \frac{1}{4 d(x, p)} \geq 160 \bar{c}(\bar{c}+1) s / 4>\bar{c} s,
$$

contradicting $\operatorname{diam}\left(X \backslash\{p\}, d_{p}\right) \leq \bar{c} s$.

Now let $\mathcal{D}$ be the subfamily of $\mathcal{C}$ consisting of the maximal elements of $\mathcal{C}$ with respect to inclusion. Then $\mathcal{D}$ is a covering of $A_{s}$. Since $\mathcal{B}=\bigcup_{k=0}^{n} \mathcal{B}_{k}$, we have $\mathcal{D}=\bigcup_{k=0}^{n} \mathcal{D}_{k}$, where $\mathcal{D}_{k}=\mathcal{D} \cap \mathcal{B}_{k}$.

Lemma 4.6. Let $C_{x} \neq C_{y} \in \mathcal{D}_{k}$ for some $k$. Then $d_{p}\left(x^{\prime}, y^{\prime}\right)>s$ for every pair $x^{\prime} \in C_{x}, y^{\prime} \in C_{y}$.

Proof. Suppose there are $x^{\prime} \in C_{x}, y^{\prime} \in C_{y}$ such that $d_{p}\left(x^{\prime}, y^{\prime}\right) \leq s$. We may assume $j(x) \leq j(y)$. Since $C_{x}$ and $C_{y}$ are both maximal elements of $\mathcal{C}$, properties (i) and (iii) of Proposition 2.2 imply that $d\left(w_{1}, w_{2}\right) \geq r^{j(x)}$ for all $w_{1} \in C_{x}$ and $w_{2} \in C_{y}$. In particular, $d\left(x^{\prime}, y^{\prime}\right) \geq r^{j(x)}$. By the definition of $j(x)$ we have $\operatorname{diam}\left(C_{x}, d_{p}\right) \leq \bar{c} s$. Let $z \in C_{x} \cup\left\{y^{\prime}\right\}$. Then $d_{p}(x, z) \leq(\bar{c}+1) s$. Since $d_{p}(x, z) \geq \frac{d(x, z)}{4 d(x, p) d(z, p)}$ and $x \in A_{s}$, we obtain $d(x, z) \leq \frac{1}{40 \bar{c}} d(z, p)$. It follows that $d(z, p) \leq 2 d(x, p)$ and so $d(x, z) \leq \frac{1}{20 \bar{c}} d(x, p)$. The triangle inequality implies

$$
d\left(x^{\prime}, p\right) \leq\left(1+\frac{1}{20 \bar{c}}\right) d(x, p) \leq 11 d(x, p) / 10,
$$

$d\left(x^{\prime}, y^{\prime}\right) \leq \frac{1}{10 \bar{c}} d(x, p)$ and

$$
d\left(y^{\prime}, p\right) \leq d\left(y^{\prime}, x^{\prime}\right)+d\left(x^{\prime}, p\right) \leq 12 d(x, p) / 10 .
$$

By property (ii) of Proposition 2.2, there is some $C^{\prime} \in \mathcal{B}^{j(x)+1}$ with $B\left(x, r^{j(x)+1}\right)$ $\subset C^{\prime}$. Let $w \in C^{\prime}$. Then

$$
d(x, w) \leq c r^{j(x)+1}=c r \cdot r^{j(x)} \leq c r \cdot d\left(x^{\prime}, y^{\prime}\right) \leq \frac{c r}{10 \bar{c}} d(x, p) \leq d(x, p) / 10,
$$

and so $\frac{9}{10} d(x, p) \leq d(w, p) \leq \frac{11}{10} d(x, p)$. It follows from property (i) of Proposition 2.2 that

$$
d_{p}(x, w) \leq \frac{d(x, w)}{d(x, p) d(w, p)} \leq \frac{c r^{j(x)+1}}{9 d^{2}(x, p) / 10} \leq \frac{c r \cdot d\left(x^{\prime}, y^{\prime}\right)}{9 d^{2}(x, p) / 10}
$$


Using

$$
\frac{d\left(x^{\prime}, y^{\prime}\right)}{4 d\left(x^{\prime}, p\right) d\left(y^{\prime}, p\right)} \leq d_{p}\left(x^{\prime}, y^{\prime}\right) \leq s
$$

$d\left(x^{\prime}, p\right) \leq 11 d(x, p) / 10$ and $d\left(y^{\prime}, p\right) \leq 12 d(x, p) / 10$, we obtain $d_{p}(x, w) \leq 8$ crs for all $w \in C^{\prime}$. It follows that $\operatorname{diam}\left(C^{\prime}, d_{p}\right) \leq 16 \mathrm{crs}=\bar{c} s$, contradicting the definition of $j(x)$.

Lemma 4.6 implies that each $\mathcal{D}_{k}$ has $s$-multiplicity at most 1 . Let $B_{s}$ be the complement of $A_{s}$ in $X \backslash\{p\}$. If $B_{s}=\emptyset$, then the proof of Proposition 4.3 is complete since $\mathcal{D}$ is $\bar{c} s$-bounded. Suppose $B_{s} \neq \emptyset$. Using

$$
d_{p}(y, z) \leq \frac{d(y, z)}{d(y, p) d(z, p)} \leq \frac{1}{d(y, p)}+\frac{1}{d(z, p)}
$$

we see that

$$
\operatorname{diam}\left(B_{s}, d_{p}\right) \leq 2 \cdot 160 \bar{c}(\bar{c}+1) s=320 \bar{c}(\bar{c}+1) s .
$$

Let $\mathcal{D}_{0}^{\prime}=\left\{C \in \mathcal{D}_{0}: d_{p}\left(C, B_{s}\right)>s\right\}, \mathcal{D}_{0}^{\prime \prime}=\left\{C \in \mathcal{D}_{0}: d_{p}\left(C, B_{s}\right) \leq s\right\}$, and set $E=B_{s} \cup \bigcup_{C \in \mathcal{D}_{0}^{\prime \prime}} C$. Then

$$
\operatorname{diam}\left(E, d_{p}\right) \leq \bar{c} s+s+320 \bar{c}(\bar{c}+1) s+s+\bar{c} s \leq 322 \bar{c}(\bar{c}+1) s<c^{\prime} s
$$

Define $\mathcal{E}_{0}=\{E\} \cup \mathcal{D}_{0}^{\prime}$, and $\mathcal{E}_{k}=\mathcal{D}_{k}$ for $1 \leq k \leq n$. Now $\bigcup_{k=0}^{n} \mathcal{E}_{k}$ is a $c^{\prime} s$-bounded covering of $\left(X \backslash\{p\}, d_{p}\right)$ such that each $\mathcal{E}_{k}$ has $s$-multiplicity at most 1 .

This completes the proof of Proposition 4.3.

\section{REFERENCES}

[A] P. Assouad, Sur la distance de Nagata, C. R. Acad. Sci. Paris Sér. I Math. 294 (1982), no. 1, 31-34. MR651069 (83b:54034)

[B] S. Buyalo, Asymptotic dimension of a hyperbolic space and the capacity dimension of its boundary at infinity, St. Petersburg Math. J. 17 (2006), no. 2, 267-283. MR2159584 (2006d:31009)

[BDLM] N. Brodskiy, J. Dydak, M. Levin, A. Mitra, Hurewicz Theorem for Assouad-Nagata dimension, preprint.

[BHX] S. Buckley, D. Herron, X. Xie, Metric space inversions, quasihyperbolic distance, and uniform spaces, to appear in Indiana University Math. Jour.

[BK] M. Bonk, B. Kleiner, Rigidity for quasi-Möbius group actions, J. Differential Geom. 61 (2002), no. 1, 81-106. MR1949785 (2004b:53059)

[BL] S. Buyalo, N. Lebedeva, Dimension of locally and asymptotically self-similar spaces, preprint. MR2319510

[BS] S. Buyalo, V. Schroeder, A product of trees as universal space for hyperbolic groups, preprint.

[D] A. Dranishnikov, On hypersphericity of manifolds with finite asymptotic dimension, Trans. Amer. Math. Soc. 355 (2003), no. 1, 155-167. MR1928082 (2003g:53055)

$[\mathrm{H}] \quad$ J. Heinonen, Lectures on analysis on metric spaces, Universitext. Springer-Verlag, New York, 2001. MR1800917 (2002c:30028)

[LS] U. Lang, T. Schlichenmaier, Nagata dimension, quasisymmetric embeddings, and Lipschitz extensions, Int. Math. Res. Not. 2005, no. 58, 3625-3655. MR2200122 (2006m:53061) 
[V] J. Väisälä, Quasimöbius maps, J. Analyse Math. 44 (1984/85), 218-234. MR801295 (87f:30059)

[V2] J. Väisälä, The free quasiworld. Freely quasiconformal and related maps in Banach spaces, Quasiconformal geometry and dynamics (Lublin, 1996), 55-118, Banach Center Publ., 48. MR1709974 (2000h:58017)

Department of Mathematical Sciences, Georgia Southern University, Statesboro, Georgia 30460

E-mail address: xxie@georgiasouthern.edu 